\title{
Killer combination
}

Combination chemotherapy is used to treat most cancers, but the drugs involved are usually selected by trial-and-error analysis. Researchers can now, however, use geneexpression profiling to assess the interaction of anticancer agents, and use this information to optimize combination therapy.

Gene-expression analysis has been useful in identifying the specific targets of many cancer drugs, but there have been no studies to compare the effects of single agents versus combinations. In the May issue of Nature Genetics, Meyling Cheok et al. analysed the effects of combination chemotherapy on the expression pattern of more than 9,600 genes in patients with acute lymphoblastic leukaemia (ALL). They studied leukaemia cells isolated from 60 individuals with newly diagnosed ALL, before and after treatment with methotrexate and mercaptopurine. These drugs exert their cytotoxic effects by different mechanisms, and high-dose and low-dose methotrexate also have different mechanisms. Patients therefore received either mercaptopurine alone, high-dose methotrexate alone, or mercaptopurine in combination with high- or low-dose methotrexate.

The authors identified 124 genes that discriminated between the four treatments. medications were given as a single agent also changed when they were given together. For example, expression of $B A X$ and $A T M$ were higher after treatment with methotrexate alone, but not in the other three treatment groups. By contrast, $E 2 F 5$ and $M L H 1$ were only upregulated after treatment with mercaptopurine alone - not in patients who received combination therapy. No genes were commonly upregulated or downregulated in all individuals across all treatments.

These findings indicate that geneexpression responses are treatment specific, and that combinations do not simply elicit the additive effects of single agents. Studies such as these should help to identify patients that are most likely to respond to different therapeutic approaches.

Kristine Novak Only $14 \%$ of genes that changed when these

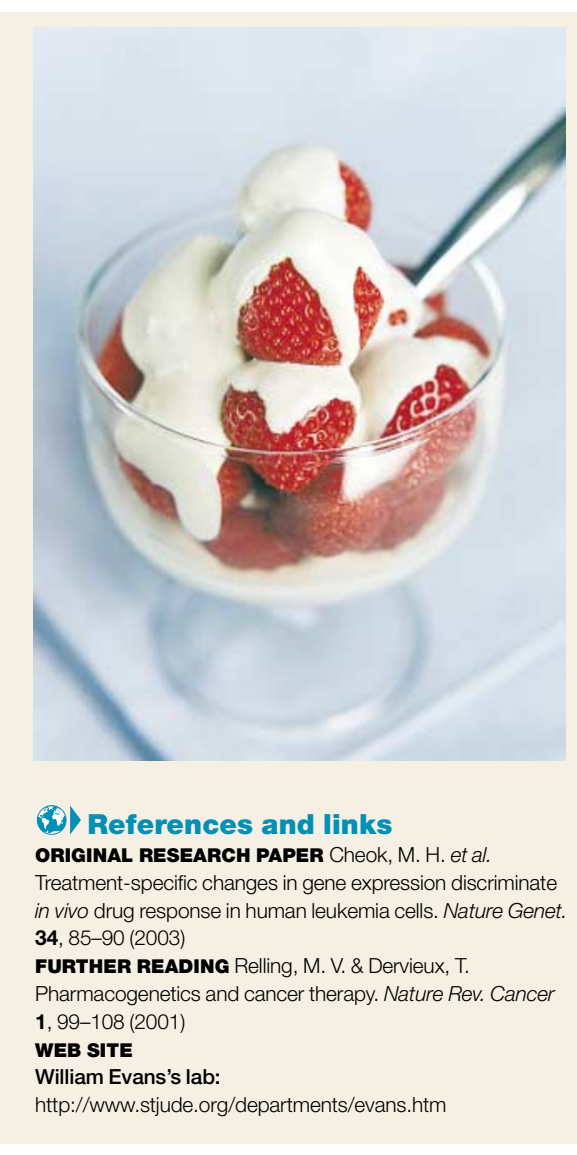

in vivo effects of YC-1 in a mouse xenograft model. Nude (immunodeficient) mice were injected with various types of tumour cell line and, once the tumour had become established, were treated daily with YC-1 or placebo. After two weeks, tumour size was significantly smaller in treated mice than in controls, irrespective of tumour type. The level of HIF- $1 \alpha$ protein was significantly lower in treated tumours, as were protein and mRNA levels of VEGF. Analysis of tumour sections showed that tumours from YC-1-treated mice had a less-well-developed vasculature and fewer $\mathrm{CD} 31^{+}$vessels (CD31 being a marker of endothelial cells).

So, it seems from this initial study that YC-1 could be used to 'suffocate' many types of tumour by preventing their ability to cope with hypoxia. Its low toxicity in the mouse study is an important advantage, although owing to its other known actions in the circulation, possible side effects in humans include increased bleeding time and hypotension.

Kirsty Minton tion. However, under conditions of hypoxia, hydroxylation is inhibited, with the end result that HIF- $1 \alpha$ is not degraded. Biologically active HIF- $1 \alpha$ increases the level of expression of VEGF.

In vitro results showed that $\mathrm{YC}-1$ inhibits the expression of HIF- $1 \alpha$ by tumour cell lines during hypoxia, so the authors tested the

\section{6) References and links}

ORIGINAL RESEARCH PAPER Yeo, E.-J. et al. YC-1: a potential anticancer drug targeting hypoxia-inducible factor 1 J. Natl Cancer Inst. 95, 516-525 (2003)

FURTHER READING Kerbel, R. \& Folkman, J. Clinical 727-739 (2002) translation of angiogenesis inhibitors. Nature Rev. Cancer 2 , 